\title{
MONITORAMENTO DA VARIAÇÃO DA CONCENTRAÇÃO DE SÓLIDOS EM ENSAIOS DE SEDIMENTAÇÃO GRAVITACIONAL PSEUDOPLÁSTICOS \\ UTILIZANDO FLUIDOS
}

\author{
F. M. FAGUNDES ${ }^{1}$, B. A. MOREIRA ${ }^{1}$, F. O. AROUCA $^{1}$ e J. J. R. DAMASCENO ${ }^{1}$ \\ ${ }^{1}$ Universidade Federal de Uberlândia, Departamento de Engenharia Química \\ E-mail para contato: flaviamfagundes@gmail.com
}

\begin{abstract}
RESUMO - A compreensão do comportamento de materiais particulados sedimentando em fluidos pseudoplásticos é de fundamental importância em diversas operações industriais, tais como, durante os processos exploratórios de petróleo e gás. Neste contexto, este trabalho teve como objetivo, o estudo da sedimentação de partículas em fluidos não-newtonianos pseudoplásticos. $\mathrm{O}$ monitoramento da sedimentação dos materiais sólidos foi conduzido em ensaios de sedimentação gravitacional em batelada. Para a execução dos testes foram utilizadas soluções nãonewtonianas preparadas à base de água e goma xantana. A técnica de atenuação de raios gama utilizada neste estudo permitiu a realização de medidas indiretas da concentração local de sólidos em função do tempo. Os resultados obtidos apresentaram novas informações em um assunto na literatura em que diversas questões ainda precisam ser exploradas e melhor compreendidas.
\end{abstract}

\section{INTRODUÇÃO}

A sedimentação é uma das operações unitárias mais utilizadas na indústria química. Esta operação consiste na separação de uma suspensão sólido-líquido. Segundo Arouca (2007), este fenômeno pode ter sua representação através das equações da continuidade e do movimento para ambos os constituintes, sólido e líquido, e pelo conhecimento de equações constitutivas relativas a cada sistema em particular. O conhecimento da concentração de partículas, por sua vez, é de extrema importância para a descrição do fenômeno.

Segundo Kynch (1952) este fenômeno é unidimensional e apresenta como característica principal o aumento da concentração com o tempo no sentido do fundo do recipiente. A teoria apresenta ainda a descrição de quatro regiões distintas do fenômeno da sedimentação em batelada: região de líquido clarificado, região de sedimentação livre, região de transição e região de formação do sedimento, separadas por fronteiras móveis que variam com o tempo e que, por sua vez, aumentam o grau de complexidade da modelagem do fenômeno. 
Teste de sedimentação em batelada é o ponto de partida para o projeto de sedimentadores em unidades industriais contínuas. Tal procedimento é realizado com base no conhecimento da concentração inicial do fluido, a fim de se obter as distribuições de concentração de partículas nos sedimentos formados e se apresenta como a forma mais simples e eficiente para o estudo das condições operacionais de sedimentadores.

\subsection{Caracterização do fluido}

Os fluidos apresentam comportamentos diferentes de acordo com a sua reologia, que os caracteriza como fluidos newtonianos, dilatantes, pseudoplásticos ou plástico de Bingham dependendo da relação entre a tensão cisalhante aplicada no fluido e a taxa deformação (Damasceno, 2005). Na Figura 1, a seguir, é apresentada a reologia de cada um dos fluidos mencionados acima.

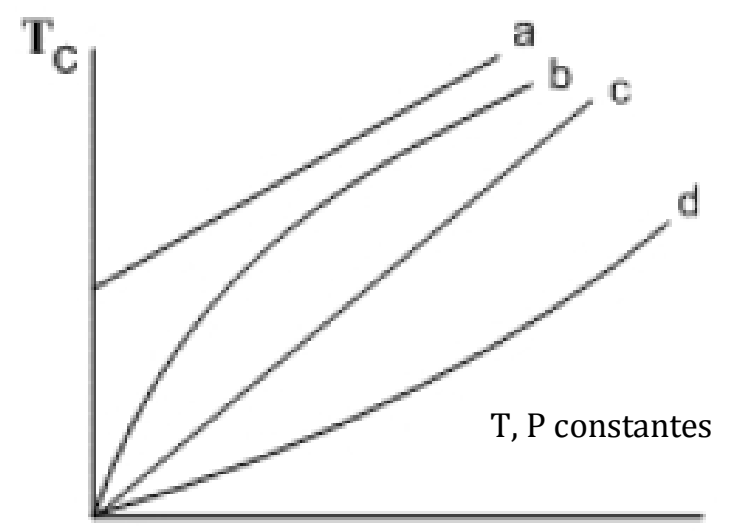

Figura 1 - Relação entre a tensão cisalhante (Tc) e a taxa de deformação (D) no fluido plástico de Bingham (a), pseudoplástico (b), newtoniano (c) e dilatante (d).

A goma xantana é um fluido não-newtoniano chamado de pseudoplástico. Segundo Borges et al. (2009), ela é produzida por espécies de bactérias do gênero Xanthomonas, normalmente Xanthomonas campestris pv campestris. Sua utilização como espessante e estabilizante tornou-se crescente com o passar dos anos, principalmente no campo petrolífero, onde é utilizada no fluido de perfuração e na recuperação terciária de poços de petróleo.

\subsection{Técnica de atenuação de raios gama}

Quando a suspensão é muito diluída, testes através de técnicas simples de amostragem podem ser realizados. No entanto, para suspensões mais concentradas deve-se optar por uma técnica que não provoque perturbações no sistema. Sendo assim, utilizar uma técnica não destrutiva baseada na medida de atenuação de raios gama é uma alternativa (Arouca, 2007). Esta técnica consiste na emissão de um feixe de radiação gama que atravessa o meio físico. Esta técnica faz a leitura indireta da concentração local de partículas. 
Trabalhos anteriores mostram a eficiência da técnica de atenuação de raios gama para caracterizar sistemas sólido-líquidos. Um desses trabalhos foi o de Ruiz et al. (1997) que aplicaram a técnica de atenuação de raios gama no estudo do processo de sedimentação em batelada a fim de mapear as concentrações de sólidos como função da posição e do tempo durante ensaios em proveta. Os resultados obtidos permitiram observar as quatro regiões apresentadas por Kynch (1952).

Outro trabalho utilizando a mesma técnica é o estudo realizado por Arouca e Damasceno (2005a) no qual usaram caulim em testes de sedimentação em batelada. Os autores admitiram a concentração local como uma função da curva de atenuação e aplicaram a técnica para determinação de parâmetros de equações constitutivas para pressão nos sólidos e permeabilidade do meio poroso. Outro estudo dos autores (Arouca e Damasceno, 2005b) usou a atenuação de raios gama no monitoramento de concentrações como função da posição e do tempo em testes dinâmicos de sedimentação em batelada. Os resultados obtidos pelos autores permitiram um mapeamento completo de uma coluna de sedimentação e a caracterização de regiões distintas do fenômeno. Os resultados obtidos permitem a validação de modelos matemáticos para a sedimentação unidimensional.

Dessa forma, aliando conhecimentos adquiridos em diversos trabalhos que têm sido publicados na literatura com a parte experimental que foi desenvolvida, foram obtidas novas informações sobre a sedimentação do fluido não-newtoniano e pseudoplástico feito de goma xantana.

\section{METODOLOGIA}

O sistema experimental foi desenvolvido no Laboratório de Separação da Faculdade de Engenharia Química da Universidade Federal de Uberlândia- FEQUI/UFU.

Para a realização dos testes foram utilizadas soluções de goma xantana a 0,2\%. A solução foi preparada adicionando no agitador em hélice com 1600 rpm, modelo RW20DZM.n da Kika Labortechnik, $1000 \mathrm{ml}$ de água destilada, $2 \mathrm{~g}$ de goma xantana, cedida pela CENPES/Petrobrás, e 2 $\mathrm{ml}$ de formaldeído até o momento em que foi percebido que o soluto havia sido dissolvido completamente.

A adição do formaldeído era necessária para impedir a proliferação de microrganismos. Desta forma era possível utilizar a solução por alguns dias sem que houvesse alteração nas características reológicas do fluido. Após o preparo das soluções, se deixava as mesma em repouso por 24 horas para a hidratação do polímero.

A Figura 2, a seguir, mostra a caracterização da solução utilizada utilizando um reômetro Brookfield R/S Plus. 


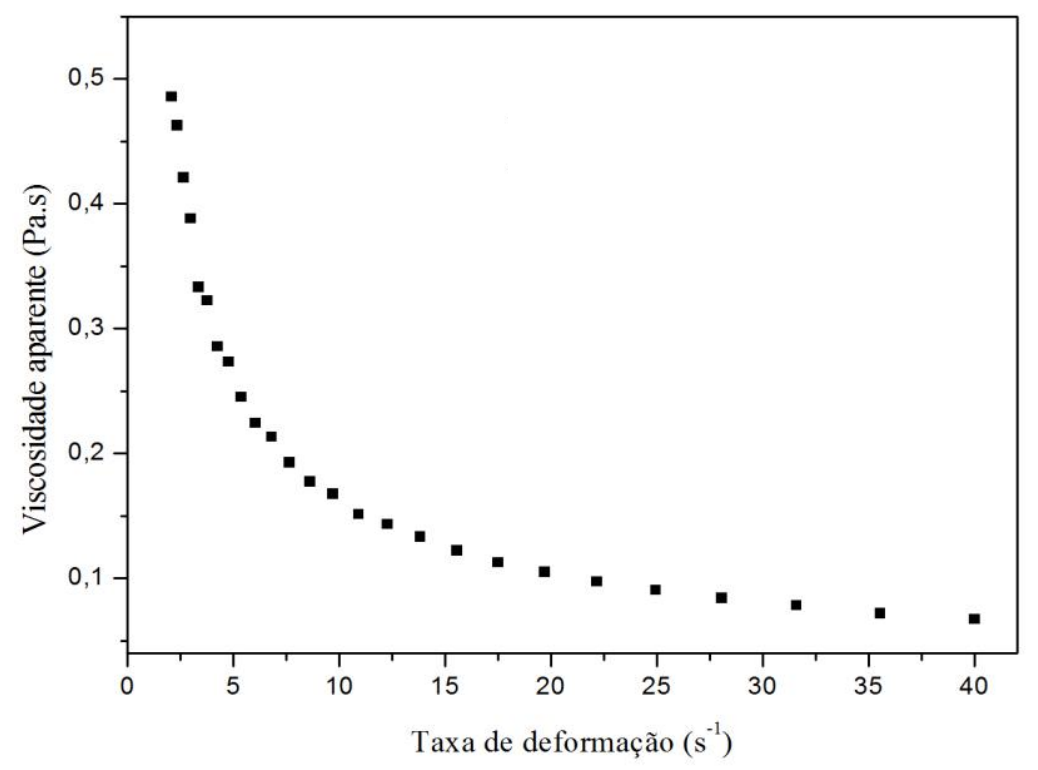

Figura 2 - Gráfico que caracteriza a solução de goma xantana $0,2 \% \mathrm{~m} / \mathrm{m}$ utilizada durante o experimento.

Como materiais sólidos foram utilizadas amostras de carbonato de cálcio. Tais amostras foram peneiradas entre as seguintes faixas: $-d_{\#}=212 \mu \mathrm{m},+d_{\#}=150 \mu \mathrm{m}\left(-d_{\#}\right.$ e $+d_{\#}$ indicam respectivamente o diâmetro de abertura da peneira no qual o sólido foi passante e retido).

\subsection{A unidade experimental}

A metodologia desse trabalho foi baseada na execução da técnica não destrutiva de atenuação de raios gama.

Em resumo, essa técnica utilizada consistiu basicamente em: (i) um feixe de radiação, proveniente de um cilindro de amerício (Am241), foi direcionado transversalmente de modo que atravesse o recipiente de testes, no qual estava contida a solução homogeneizada a ser analisada, e fosse captado por uma válvula fotomultiplicadora, integrante ao sistema de detecção. À medida que este feixe chegou ao sistema de detecção, foi realizada a leitura indireta da concentração local através de um computador; (ii) tal procedimento foi repetido diversas vezes para a mesma solução em diferentes posições verticais.

A Figura 3 apresenta um esquema geral da unidade experimental de aplicação de radioisótopos utilizada por este trabalho. 


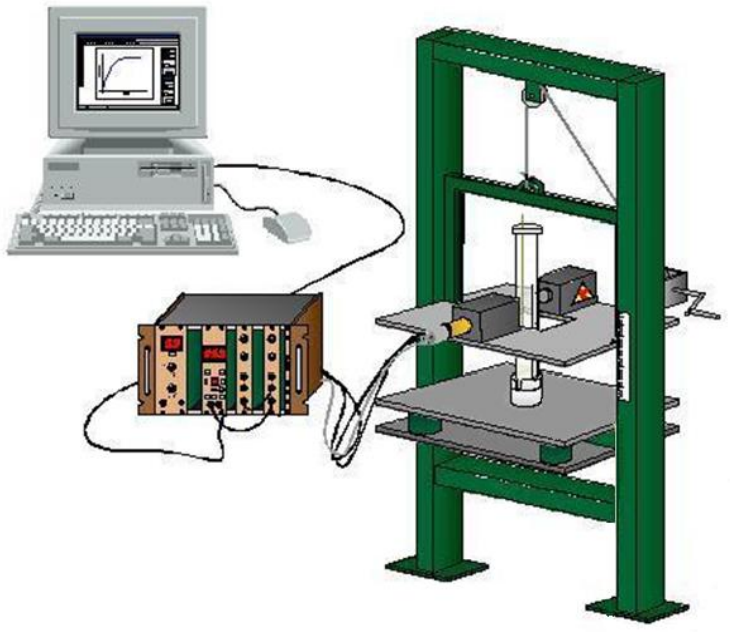

Figura 3 - Esquema da unidade experimental utilizada para ensaio de sedimentação utilizando técnica de atenuação de raios-gama.

\subsection{Monitoramento da variação de concentração de sólidos ao longo do tempo para diferentes posições da proveta de testes}

Com a solução de goma xantana preparada se adicionava do carbonato de cálcio de modo que a concentração volumétrica de sólidos inicial da suspensão ficasse em $\varepsilon_{s}=4 \% \mathrm{v} / \mathrm{v}$.

Em seguida, a suspensão era homogeneizada manualmente no eixo vertical com um misturador cônico vazado cuja base apresenta diâmetro levemente menor do que o recipiente de testes. Com a suspensão homogeneizada se iniciava o monitoramento da variação de concentração de sólidos ao longo do tempo por meio da técnica de atenuação de raios gama.

\section{RESULTADOS}

A partir do experimento de sedimentação em batelada da solução de goma xantana e da utilização da técnica de atenuação de raios gama foi possível obter as distribuições de concentrações volumétrica, formadas pela sedimentação de partículas sólidas de carbonato de cálcio, como se pode notar na Figura 4. 


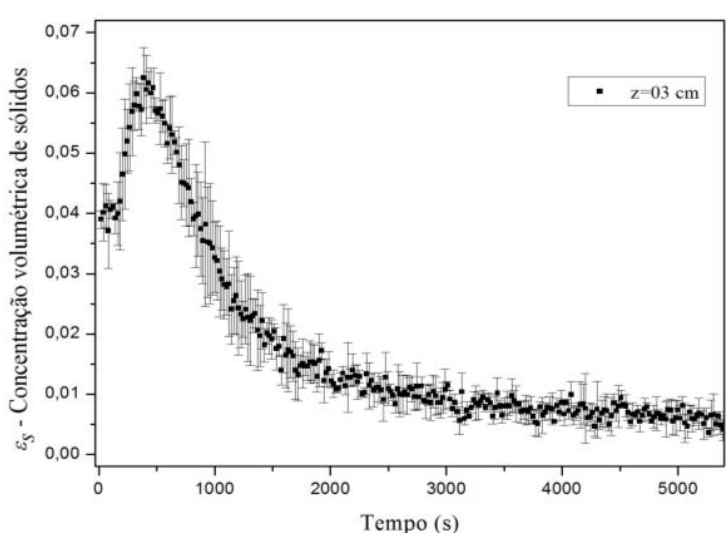

(a) Posição $\mathrm{z}=3 \mathrm{~cm}$

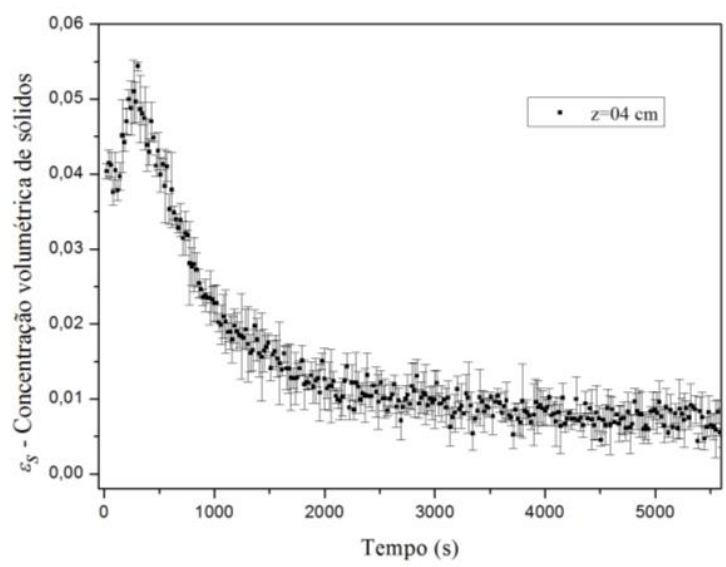

(b) Posição $\mathrm{z}=4 \mathrm{~cm}$

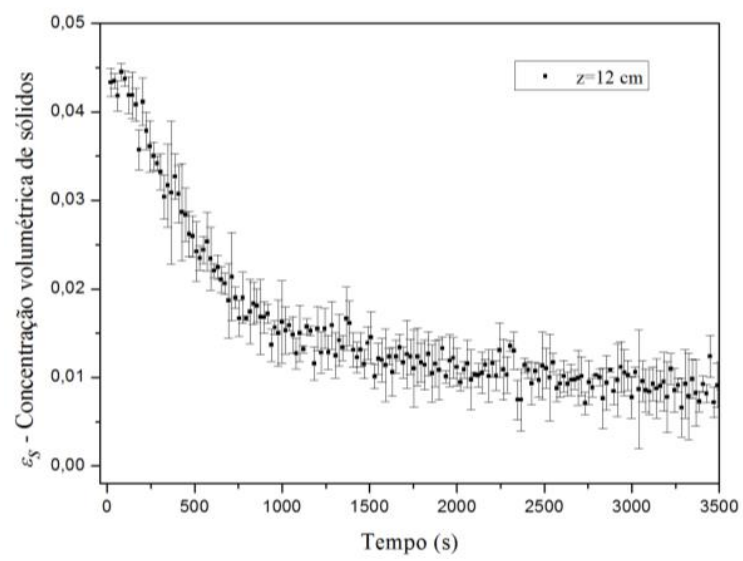

(c) Posição $\mathrm{z}=12 \mathrm{~cm}$

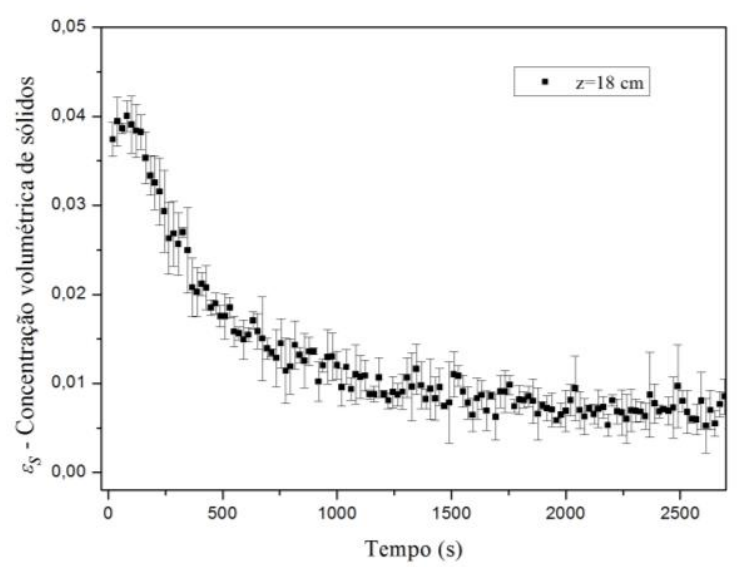

(c) Posição $\mathrm{z}=12 \mathrm{~cm}$

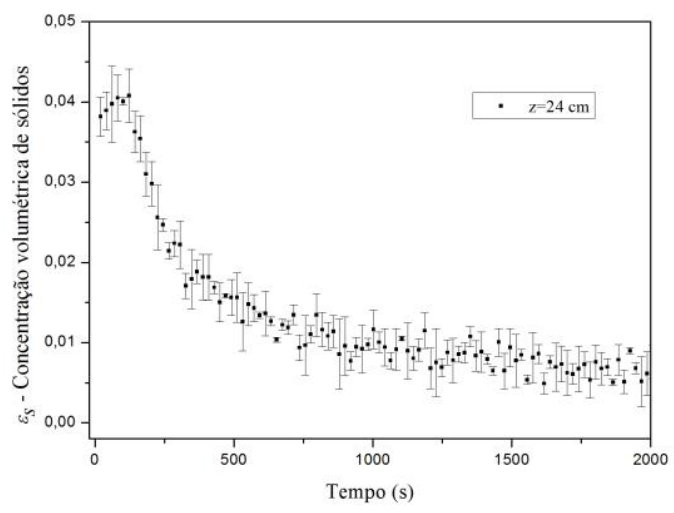

(e) Posição z=24 cm

Figura 4 - Distribuição de concentrações nas posições: $3 \mathrm{~cm}(\mathrm{a}), 4 \mathrm{~cm}$ (b), $12 \mathrm{~cm}$ (c), $18 \mathrm{~cm}$ (d) e $24 \mathrm{~cm} \mathrm{(e),} \mathrm{sendo} \mathrm{z}$ a altura medida a partir da base da proveta em que $\mathrm{z}=0$. 
Ao se comparar os resultados clássicos de sedimentação em fluidos newtonianos com os resultados deste trabalho se pode avaliar, como principal diferença, a maneira como ocorreu a passagem da interface clarificada diante do sistema de medidas.

No caso da sedimentação no fluido pseudoplástico, se verificou que a diminuição na concentração de sólidos ocorreu de maneira rápida inicialmente e depois foi reduzindo sua velocidade ao longo do tempo. Como consequência deste fenômeno, não houve uma brusca redução da concentração em um curto período de tempo, como comumente ocorre no caso de fluidos newtonianos (Kynch, 1952; Ruiz 1997 e Arouca 2007). Uma explicação para tal fenômeno pode ser atribuída aos mecanismos de segregação de tamanhos entre as partículas que é mais relevante em fluidos pseudoplásticos.

Nas Figuras 4a e 4b, foi possível verificar o aumento da concentração de sólidos no início do ensaio antes de ocorrer a diminuição de concentração até tender a zero. Este aumento verificado pode ser explicado pela região de transição entre a formação do sedimento e a região de sedimentação. Nela, nota-se que inicialmente que a concentração permanece constante por um determinado tempo, depois ocorreu um aumento da concentração de sólidos de 4 até aproximadamente 6,5\% na Figura 4a e apresentou valores próximos de 5,5\% para a Figura 4b. A partir de então, foi observado o decréscimo até valores próximos de zero.

No entanto, nas Figuras 4c a 4e, foi observado que a variação da concentração não apresentou uma aumento na concentração de sólidos antes de iniciar a diminuição da concentração que se aproximou de zero, caracterizando essas alturas como pertencentes a região de líquido clarificado.

\section{CONCLUSÃO}

Com base no estudo teórico e experimental da sedimentação gravitacional em batelada, foi possível verificar a eficácia da técnica de atenuação de raios gama na determinação da distribuição da concentração volumétrica de amostras de carbonato de cálcio em soluções de goma xantana.

O comportamento das partículas sedimentando no fluido pseudoplástico, se mostrou diferente dos resultados conhecidos de sedimentação em fluidos newtonianos. A principal diferença verificada foi na maneira com a qual ocorre a passagem da interface clarificada.

Foi possível constatar também a existência de uma região de concentração variável na sedimentação em goma xananta. Este aumento verificado pode ser explicado pela região de transição entre a formação do sedimento e a região de sedimentação.

\section{REFERÊNCIAS}


AROUCA, F. O.; DAMASCENO, J. J. R. Concentration profiles and iso-concentration curves for batch settling using the gamma rays attenuation technique. Fifth International LatinAmerican Conference on Powder Technology - PTECH, 2005a.

AROUCA, F. O.; DAMASCENO, J. J. R. Analysis of the behavior of solid-liquid systems based on the shape, size distribuition and density of the solid particles. Proceedings of Fifth International Latin-American Conference on Powder Technology - PTECH, 2005b.

AROUCA, F. O. Uma Contribuição ao Estudo da Sedimentação Gravitacional em Batelada. Tese (Doutorado) - FEQ/UFU, 2007.

BORGES, C. D.; VEndRUSCOlO, C. T.; MARTINS, A. L.; LOMBA, R. F. T. Comportamento reológico de xantana produzida por Xanthomonas arboricola pv pruni. Polímeros: Ciência e Tecnologia, vol. 19, nº 2, p. 160-165, 2009.

DAMASCENO, J. J. R. Apostila usada para ministrar aulas da disciplina fenômenos de transporte. Graduação da Engenharia Química. FEQ/UFU, 2005.

KYNCH, G. J. A theory of sedimentation. Trans. Amer. Soc., p. 166-176, 1952.

RUIZ, M. I.; FERNANDES, R. S.; DAMASCENO, J. J. R. Mapeamento de concentração de sólidos em função da posição e do tempo para ensaios de sedimentação em batelada. $X X V$ Congresso Brasileiro de Sistemas Particulados, 1997. 